\title{
Theorizing Corruption through Agent-Based Modeling
}

\author{
Hokky Situngkir ${ }^{1}$ Deni Khanafiah ${ }^{2}$ \\ ${ }^{1}$ Dept. Computational Sociology, Bandung Fe Institute, mail: hs@compsoc.bandungfe.net \\ ${ }^{2}$ Scholar, Bandung Fe Institute, mail: dk@students.bandungfe.net
}

\begin{abstract}
The aim of this paper is to gain the broad explanation of corruption using simple computational model. We elaborated further the model of corruption described previously in [4], with some additions in model's properties. We performed hundreds of experiments computationally using Swarm and constructed the explanation of corruption based upon these results. We show that corruption should be understood as complex social-phenomena, which relates not only with economical aspect, but also with many other social and anthropological aspects.
\end{abstract}

Keywords: corruption, agent-based model, Swarm.

\section{The Social Structure of Corrupt Behavior}

In many cases, corruption is a signifier referring to many phenomena. Most of literatures on corruption refer to many phenomena i.e.: bribery, collusion, nepotism, and so on. According to [1], corruption is the abuse of public power for private benefit, while the private benefit is often in the form of illicit money or in-kind from a client to the agent; we call this as bribery. Conclusively, the evaluation of the definition on corruption from many literatures evokes us to realize that the terminology of corruption is a floated signifier many terminologies should have point out.

Thus, we can say that corruption is a symptom of the weakness of political, social, legal and economic systems. Even where corruption is widespread, the actor will strive to keep it hidden from public view. Corruption is not new, nor is it confined to any particular part of the world. On the contrary, corruption is a global phenomenon, although its severity varies from country to country.

One important thing to note is that the different definitions on corruption eventually impact the way we measure and analyze a corrupt phenomena. [1] analyzed some different methods on measurement on corruption. Today, corruption is measured through surveys and polls of random sample from citizens or businesspeople; this is what can be called the method of subjective measures, although each of the subjective measurement employ distinguishable methodologies: the first based on the perceptions and the other based on experience. However, the paper will not discuss the methodologies of the measurement since the purpose of the paper is to find some general structural outline of corruption gained through computational simulations.

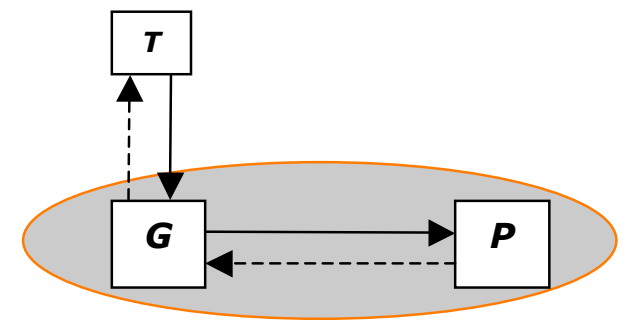

Fig. 1. The primitive model of corruption. The solid arrows represent things to be done according to rule of the game and the other represent situation when corruption occurs.

To define what corruption is, we must recognize the social agent that involved in the process. According to [2], the types of agent in corrupt behavior i.e.: there are at least three elements involved in the corruption: element (can be individual, such as an employer, or a collective body) relying on the expectation that people in certain positions are bound to follow given rules, agent who agrees to act on behalf of the first element, and the other agent whose interests are affected by the first agent's actions. There are rules the second element must obey as trusted by the first one. However, the second agent wants the first one to do improperly: to abuse the first agent's trust from the first elements in order to gain benefit for her. For the sake of easier linguistic environment we will give one element and two types of agent here; the first element is member of the set $\boldsymbol{T}$, to give trust to the first agent. The first agent is member of the set $\boldsymbol{G}$ receiving obligation to play according to rules that tie $\boldsymbol{G}$ and $\boldsymbol{T}$. However, during the process there will be the second 
agent, the member of the set $\boldsymbol{P}$, try to attract member of $\boldsymbol{G}$ to violate the rule specified. Once member of $\boldsymbol{G}$ agrees to do it, the corruption on abusing the authority occurs. We should note that a corruption can only occur if and only if a certain relationship between members of $\boldsymbol{T}$ and members of $\boldsymbol{G}$ pre-exists.

In micro situation, the abuse of trust given to government agent, $\boldsymbol{G}$, in her service to citizens, $\boldsymbol{P}$, will compel citizens to pay more $(\boldsymbol{y})$ in order to get her rights as it has been stated literally in the law $(\boldsymbol{x})$, otherwise there will be reduction on what citizens should get.

An act of corruption is then defined by the amount of some currency, say $\boldsymbol{b}^{*}$, received by member of $\boldsymbol{G}$ as compelled by member of $\boldsymbol{G}$ or persuaded by the member of $\boldsymbol{P}$. This will make members of $\boldsymbol{G}$ abuse the trust they received. We can say that the value of $\boldsymbol{b}^{*}$ to fulfills

$\boldsymbol{y}+\boldsymbol{b}^{*}>\boldsymbol{x}$

This is the value that motivates members of $\boldsymbol{G}$ to abuse the trust they received. Member of $\boldsymbol{P}$ can vary the value of $\boldsymbol{b}^{*}$, and in return the members of $\boldsymbol{G}$ obviously can reject the offer because it is too small relative to the cost they will incur by obeying the rule or probably because their integrity: moral values keep them not to abuse the trust. It is important to note that there should be an exchange or agreement between members of $\boldsymbol{P}$ and $\boldsymbol{G}$ for each possible corrupt action.

\section{The Computational Dynamics of Corruption}

Thus, we build two set of agents, representing members of $\boldsymbol{G}$ and members of $\boldsymbol{P}$. Each agent will interact in the trust game based on the pre-defined payoff matrix. The agents will have the neighbors who are chosen randomly from the member of each set. At each round of game, each agent of $\boldsymbol{P}$ will choose the corruption partner from the collection of members of $\boldsymbol{G}$ randomly, and will have a payoff based on the action chosen by her and her partner, whether it to corrupt or not corrupt (figure 1). As explained above, corruption itself will occur only when two partnership agents agree to do corrupt.

In the simple model, as adapted from [3] to choose whether to corrupt or not to, every agent will consider two subjective considerations, they are:

- Subjective thought whether or not her partner will agree to corrupt in the next round, denoted by

$F=$ match corrupt partners/memory
- Subjective thought whether or not she will be caught, denoted by $C$, $C=$ Friends in jail/corrupt friends

while the payoff matrix is described in the following table:

\begin{tabular}{|c|c|c|}
\hline & Corrupt & Not Corrupt \\
\hline Corrupt & $\alpha$ & $\beta$ \\
\hline Not Corrupt & $\beta$ & $\beta$ \\
\hline
\end{tabular}

In our toy model, we also gave each agent the honesty index, the index depicting how corrupt one to be. Technically, the index is (truncated) Gaussian distributed random numbers, $N(0.02, \mu)$ in the interval $[0,1]$. This variable also determines the expectation value of each agent. The closer the index to unity, the greedier the agent be,

$\alpha^{*}=(1-i) \alpha$

where the $\alpha^{*}$ is the expectation of the corruption, and $i$ is the honesty index.

To evaluate the expectation value of corruption, each agent uses the limited information which constrained by several aspects, including her social network, her memory, etc. The agent never knows and calculates the macro-state of the system. The expectation value of each agent is calculated by evaluating her neighborhood,

- From subjective thought $F$, we can see that each agent will evaluate the possibility that her partners will agree to do corruption. The agent will calculate this possibility by referring to the action of her partners in last rounds (we represent it with the memory of agent with certain length that records the last action of her partners in certain length of rounds). Clearly, the expectation value of corruption is bigger when the agents always interact with the partners who chose corrupt in the previous interactions.

- However, each agent should consider that there is possibility of being caught when she chooses to do corrupt. Each agent will evaluate this possibility by calculating the recent value of $C$. The expectation of the corruption of one agent is bigger, when there are many friends of her doing corrupt but only few go to jail; the smaller of value of $C$ the bigger expectation of the agent is.

- Beside that, the agent should also consider that she will lose the not-corrupt payoff $(\beta)$ for certain of $k$ rounds when she is caught and sentenced into jail. In this case we can consider $k$ as the length of jail term. The agent will consider the possibility of losing this payoff by evaluating how many friends got into jailed when 
they do corrupt. In other word, the expectation of the agent will also governed by the value of $C$ and the jail period ( $k$ ), the bigger of $C$ and $k$, the smaller expectation of agent.

From the explanation above, we summarize the expectation value of corruption as follows:

$$
E(x)=(1-C)\left[F \alpha^{*}+(1-F) \beta\right]+C(\beta-k \beta)
$$

If the expectation $E(x)>\beta$, which means the expectation of the corruption $E(x)$ is bigger than the expectation of not to corrupt $(\beta)$, then the agent have been greedy enough to do corruption. It is obvious that each agent builds up subjective perspectives on her environment then chooses to corrupt or not-corrupt.

\section{The Computational Simulation}

We use Swarm [5] as programming language to simulate the model, and then analyze the dynamic and evolutionary process of corruption on various numerical variables and parameters.

As discussed above, there are two types of Agent, i.e. $G$-agent that represent members of $\boldsymbol{G}$ and $P$-agent that represent members of $\boldsymbol{P}$. All agents will have a given variables, i.e. the id, randomly distributed of honesty index among agents (Gaussian distribution and truncated Gaussian distributions with certain mean), and her social network.

behavior:

The $P$-agent following certain rules as their

- The P-agent will choose one of member of unjailed G-agent as her partner, and make offer to her to do corrupt or not-to-corrupt.

- The P-Agent who chooses to corrupt will have the probability of getting busted.

- The P-agent will calculate the expectation value based on her subjective thought, honesty index and jail term as described in previous section.

- The $P$-agent changes her choice whether or not to do corruption based on her expectation value.

While the $G$-agent follows certain rules:

- The G-agent will interact with P-Agent and decides whether or not she agrees to corrupt

- The G-Agent who chooses to corrupt will have the probability of getting busted.

- The G-agent will calculate the expectation value based on her subjective thought, honesty index and jail term as described in previous section.

- The G-agent changes her action, whether or not to do corruption based on her expectation value.

One round of the game consists of the following sequential methods or schedules that executed by every agent:
- makeOffer, method for every P-agent in our system to choose the partner randomly, and then interact with them by making offer to do corrupt. From her interaction the $P$-agent will receive payoff according to her action and her partner's. The action of her partner will also be recorded in her memory.

- receiveOffer, method for every G-agent to receive an offer from $P$-agent, and decide to agree or not to do corrupt, and then receive payoff. The agents also record the action of her $P$ partner on her memory.

- calcultatedCorruptFriend, method for every agent to calculate how many corrupt agents in her social network.

- sendAgentToJail, method that make every corrupt agents (both of P-agents or G-agents) have the probability of being caught. The jailed agent should be removed from game for a certain rounds $(k)$ and cannot make an offer or receive it. Furthermore, along the jail term, she does not receive any pay-off.

- calculateExpectation, method for every agent to calculate her expectation value of the corruption

- changeTheAction, method for every agent to choose whether or not she corrupt based on the expectation value she has.

With ingredients of methods above, we observe and analyze the result of simulation as the macro properties of the system, including the number of corrupt agents at each round, the number of corrupt actions (both of partnership agents agree to corrupt), and the average number of the corrupt agent and corrupt action after 1000 rounds. We vary the variable of simulation to analyze what kind of structure that emerges from particular properties of the agent. We summarize the result of the simulation in the next section.

\section{Discussion and Simulation Result}

We do simulations to test computationally some common-sensical micro-macro linkage about corrupt behaviors and how to combat them.

As we can see in figure 2 , there are a strong effect of law-enforcement to decrease the corrupt behavior among people. The higher the probability of being caught in the social system, the lower the average numbers of corruption in it. This is contrary with the honesty index. There is only little contribution of higher honesty index to combat corrupt behavior. Somehow, this shows us that corruption still persists in the social system no matter how honest the people are as long as the law is not enforced. 

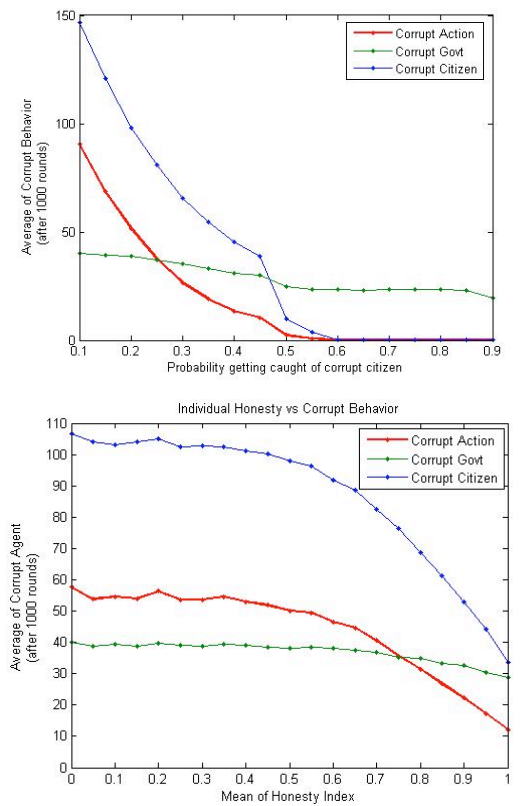

Fig. 2 effect probability of being caught for corrupt citizen on corrupt behavior (above) and simulation results for various of mean honesty index.

Another interesting result we get from the simulation is the effect of social network sizes to the corrupt behavior in the system. The social network size of agent reflect how much information in the surroundings could be gained by the agent. It also reflects indirectly the level of the democratization in our social system for the assumption that the more democratic the social system is, the more information about other agents can be gained by social agents.

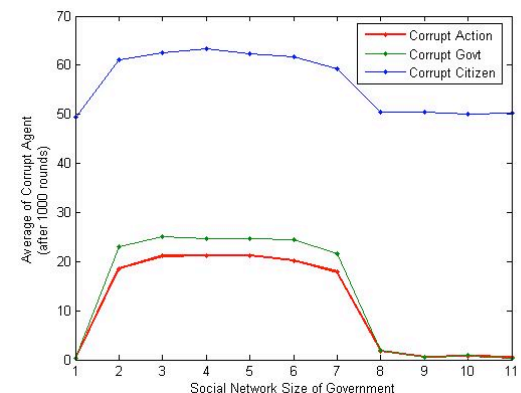

Fig. 3 The effect of the size of social network to the corrupt behavior.

There is an opinion on some people that transparency and accountability of the government will reduce the corruption as well. In our simulation for varied government social network sizes - as we can see in figure 3 - the result shows us that there are stable corrupt regime when social network is consisted of 2 up to 7 agents. Furthermore, if we add bigger social network size, the average number of corrupt actions, corrupt government and corrupt citizens decrease fast.
This shows us that there is a kind of transition as the social network size grows. As introduced before, the social network size is related to the level of ability to gain information about his surroundings as broad as possible and that in governmental system practically this can be seen as the level of democratization in the social system. Thus, there are some points that social network sizes do not give any influence to the anticorruption efforts, or in other words, there is a nonlinear relatedness between the democratization with the corrupt activities in social system.

\section{Concluding Remarks}

We have built agent-based model of corruption to capture the dynamics of corruption in social system, since the corruption phenomena itself can be seen as the macro-structure that is emerged by simple interaction among individual with particular properties at the micro level. By the computational simulation experiment, we have shown and tested some commonsensical hypothesis about corruption and how to reduce it in society. Here, we see that corruption cannot be seen mono-dimensionally. The root of corruption lies upon many social aspects, including political, economical and cultural aspects. In return, to combat corruption, we should arrange comprehensive strategies which also involving many fields of discourse. Again, it is obvious that corruption is an interdisciplinary of discussion.

\section{References}

[1] Berg, Erlend. (2001). How Should Corruption be Measured?. MSc Economics extended essay. London School of Economics and Political Science.

[2] Gambetta, Diego. (2000). "Corruption: An Analytical Map". In S. Kotkin and A. Sajo (eds.), Political Corruption of Transition: A Skeptic's Handbook, Central European University Press.

[3] Hammond, Ross. (2000). Endogenous Transition Dynamics in Corruption: An Agent-Based Computer Model. Working Paper No.19. Center on Social and Economic Dynamics. The Brookings Institution.

[4] Situngkir, H. (2003b). "The Dynamics of Corruption in Indonesia: Artificial Society Approach". Journal of Social Complexity 1(3). Bandung Fe Institute.

[5] Luna, F., \& Perrone, A. (eds.) (2002). Agentbased Methods in Economics and Finance: Simulations in Swarm. Kluwer Academic Publishers. 